Proc. of the 11 Int. School on Theoretical Physics Symmetry and Structural Properties of Condensed Matter, Rzeszów 2014

\title{
Electrons in a Semiconductor Quantum Well of the Magnetic Tunneling Structure
}

\author{
T. SZCZEPAŃSKI ${ }^{a, *}$ AND V.K. DUGAEV ${ }^{a, b}$ \\ ${ }^{a}$ Department of Physics, Rzeszów University of Technology, al. Powstańców Warszawy 6, 35-959 Rzeszów, Poland \\ ${ }^{b}$ Departamento de Física and CeFEMA, Instituto Superior Técnico, Universidade de Lisboa, \\ av. Rovisco Pais, 1049-001 Lisbon, Portugal
}

\begin{abstract}
We discuss the properties of resonant tunneling diode with resonant levels in the quantum well. The energy levels are formed inside the well as a consequence of quantization of the states between two potential barriers. We solved the Schrödinger equation for the multilayer structure and found the energy of resonant level as a function of the width of quantum well for different parameters of energy profile in the equilibrium. The results present the dependence of spin splitting in the quantum well of nonmagnetic semiconductor on the spin polarization of electrodes.
\end{abstract}

DOI: $10.12693 /$ APhysPolA.128.222

PACS: 72.25.Dc, 73.21.Fg, 85.75.Mm

\section{Introduction}

Resonant tunneling diodes $[1,2]$ attracted a lot of attention recently [3-7] due to their unique sensitivity to small variations of the external bias. The reason for such sensitivity is the presence of discrete or quasi-discrete levels in the quantum well between two tunneling junctions, so that at some energy of incoming electrons the tunneling amplitude has a very strong maximum, which can be easily registered. The resonant tunneling can be also observed in structures with a quantum dot between two metallic electrodes and double tunnel barriers on both sides of the quantum dot. However, in the case of quantum dot the problem is much more complicated because of the essential role of electron-electron interactions. Such effects are not so important for the quantum well, and the properties of systems with a twodimensional quantum well can be described in the oneparticle approximation.

The sensitivity of tunneling diodes to the location of energy levels in the quantum wells makes it possible to study the small spin splitting of the levels when the semiconductor is magnetized [8]. In the case of the quantum well in nonmagnetic semiconductor there is also a possibility of spin splitting of levels in quantum well provided that the electrodes are ferromagnetic. It is related to the difference in the flux of spin up and down electrons propagating through the quantum level.

It should be noted that the problem of spin-resolved tunneling in magnetic tunneling structures is of main importance for various spintronic applications [9]. For example, by using $\mathrm{F} / \mathrm{I} / \mathrm{F}$ structures (here $\mathrm{F}$ refers to a ferromagnet and I to an insulator), one can achieve strong tunnel magnetoresistance (TMR) [10-12] effect varying

*corresponding author; e-mail: tszczep@prz.edu.pl magnetic configurations of $\mathrm{F}$ electrodes. Besides, there appears a possibility of spin torque transfer and, correspondingly, the manipulation of the magnetic moment of a ferromagnet. Obviously, the functionality of such devices can be enhanced by including magnetic or nonmagnetic semiconductor layers into tunneling magnetic structures. An important example is a Si-based ferromagnetic structure [13-15].

In this work we consider the spin splitting of energy levels in the quantum well of nonmagnetic semiconductor in equilibrium. In such a case there is no spin-polarized current through the quantum well. However, ferromagnetic electrodes can affect the electron states in the quantum well due to the different hopping amplitudes of spinpolarized electrons through the tunneling barrier. Our work is mostly inspired by the experiments of Refs. [1619], where the spin splitting of level in the quantum well has been registered by the current-voltage characteristics and spin-resolved luminescence measurements. Our model and the choice of parameters mostly correspond to these experiments.

\section{Model}

We consider the semiconductor structure schematically presented in Fig. 1, which shows the variation of the conduction band edge along the $x$ axis. The structure consists of a $\mathrm{ZnBeSe}$ quantum well (QW) separated from ZnMnBeSe diluted-magnetic semiconductor by a thin tunneling barrier. On the other (right) side of the QW there is a relatively thick barrier. Both barriers are of $\mathrm{ZnSe}$ semiconductor. We assume that only tunneling of electron through the left very thin barrier should be taken into acount to establish the energy spectrum of electrons. The magnetization of $\mathrm{ZnMnBeSe}$ is generated by weak magnetic field which polarizes the magnetic moments of Mn atoms. This model corresponds to the experiment of Refs. $[18,19]$. 
Due to the size quantization in QW, a localized electron state with energy $E_{0}$ can be formed. Even though the $\mathrm{ZnBeSe}$ is nonmagnetic, there appears magnetic splitting of the quantization level due to the different tunneling probability through the barrier of spin up and down electrons. The spin splitting of the conduction energy band of ZnMnBeSe is defined by $U_{u}$ and $U_{d}$ for the spinup and spin-down energy bands, respectively.

Here we assume that this structure is in equilibrium, which means that there is no electric current in $x$ direction. We consider the electron states in the quantum well. In the case of magnetized electrode, one can consider separately up and down spin states corresponding to the orientation of quantization axis, which is defined by the magnetization vector. Therefore, in the following we present the results for an arbitrary position of the off-band parameter $U$, which can refer to any spin polarization. Correspondingly, we denote by the same $E_{0}$ the energy level for spin up or down electrons.

The problem should be treated in a different way for the case of $U>E_{0}$ and $U<E_{0}$. Indeed, in the first case the $E_{0}$ level is discrete but in the opposite case there is no size quantization and no pure localized states due to the penetration of an electron to the continuos spectrum of left electrode.

\section{Discrete quantum level}

In the case of $U>E_{0}$ the wave function of the localized state can be presented as

$$
\begin{array}{lc}
\psi(x)=A \mathrm{e}^{\kappa x}, & x<0, \\
\psi(x)=B \mathrm{e}^{\kappa_{0} x}+C \mathrm{e}^{-\kappa_{0} x}, & 0<x<d, \\
\psi(x)=D \mathrm{e}^{\mathrm{i} k_{0} x}+G \mathrm{e}^{-\mathrm{i} k_{0} x}, & d<x<d+a, \\
\psi(x)=F \mathrm{e}^{-\kappa_{0} x}, & x>d+a,
\end{array}
$$

with coefficients $A, B, C, D, G, F$, which should be determined by using the conditions of continuity of the the wave function and its derivative at all interfaces, as well as the usual condition of wave function normalization. Here we denoted

$$
\begin{aligned}
& \kappa=\sqrt{2 m\left(U-E_{0}\right)} / \hbar, \\
& \kappa_{0}=\sqrt{2 m\left(U_{0}-E_{0}\right)} / \hbar, \\
& k_{0}=\sqrt{2 m E_{0}} / \hbar .
\end{aligned}
$$

We assume no in-plane motion of electrons, i.e. the corresponding components of the wave vector $k_{y}, k_{z}=0$. It means that the discrete levels in this model determine the location of energy-band edges of the two-dimensional motion of spin-polarized electrons within the QW.

Using six equations for the continuity at $x=0, x-d$ and $x=d+a$, and the equation of normalization we can find the coefficients in (1)-(4) and an equation determining the energy of the localized state in the QW:

$$
\frac{\kappa_{0}\left(\mathrm{e}^{2 \kappa_{0} d}+1\right)+\kappa\left(\mathrm{e}^{2 \kappa_{0} d}-1\right)}{\kappa\left(\mathrm{e}^{2 \kappa_{0} d}+1\right)+\kappa_{0}\left(\mathrm{e}^{2 \kappa_{0} d}-1\right)}=\frac{\kappa_{0}}{k_{0}} \frac{\cos \left(k_{0} a\right) \pm 1}{\sin \left(k_{0} a\right)} .
$$

By using Eqs. (5)-(8), one can calculate the energy $E_{0}$ of the localized state. The results of numerical calculation

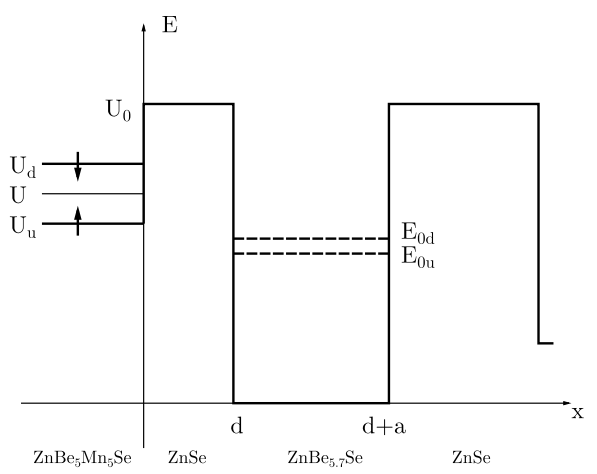

Fig. 1. Conduction band profile in the multilayer structure with a magnetized left electrode (spin injector) and electron states in the semiconductor quantum well.

of this energy as a function of parameter $U$ are presented in Fig. 2. The dependence $E_{0}(U)$ determines the spin splitting, which is the difference between two values of $E_{0}$ corresponding to $U=U_{u}$ and $U=U_{d}$, respectively. Obviously, the spin splitting is strongly depending on the thickness of tunnel barrier $d$.

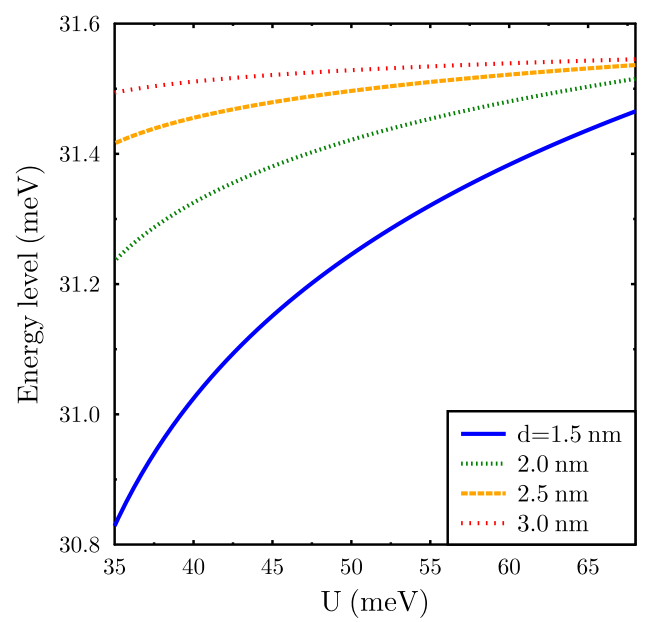

Fig. 2. Dependence of the level position $E_{0}$ on the offband parameter $U$ for different values of the barrier width $d$. The case of $E_{0}<U$.

\section{Quasi-discrete level}

In the case of $U<E_{0}$ there is an electron tunneling from the level into conduction band of $\mathrm{ZnBeMnSe}$. Thus, the level is quasi-discrete because there are electron states with all energies $E>U$. In this case, one can calculate the distribution $|\psi|^{2}(E)$ where $\psi$ is the wave function inside the QW (for $x=d+a / 2$ ). The peak at this 


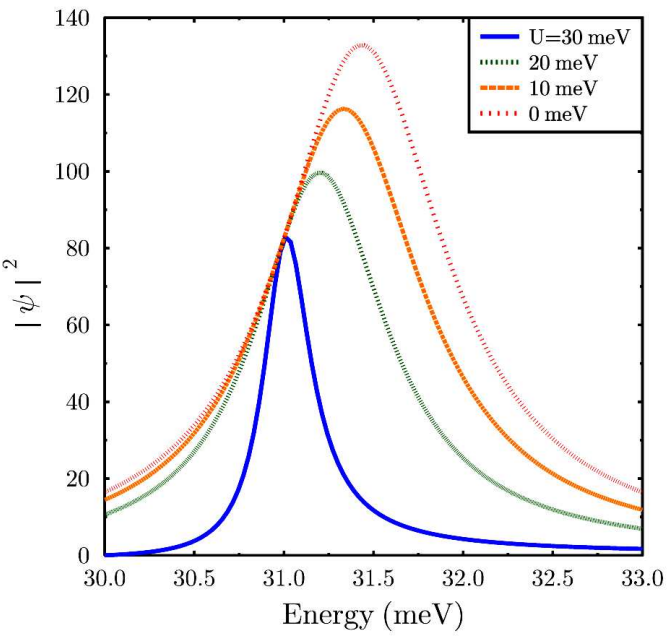

Fig. 3. Probability distribution $|\psi|^{2}(E)$ for different values of the off-band parameter $U$ in the case of $U<$ $E_{0}$. The maximum of each curve corresponds to the position of quasi-discrete electron energy level.

dependence on the energy corresponds to the position of quasidiscrete level in QW. The maximum of each curve goes to higher $E$ as $U$ goes down.

For $x<0$ the wave function is

$$
\psi(x)=\mathrm{e}^{\mathrm{i} k x}+A \mathrm{e}^{-\mathrm{i} k x}, \quad x<0,
$$

with $k=\sqrt{2 m(E-U)} / \hbar$, whereas for $x>0$ it has the same form as in Eqs. (2)-(4) with substitution $E_{0} \rightarrow$ $E$. Using Eqs. (2)-(4), (9) and the continuity condition for the wave function and its derivative at all interfaces, we can find the coefficients $D$ and $E$ determining the behaviour of $\psi(x)$ within the quantum well for any values of energy $E$. The relation between coefficients $G$ and $D$ is

$$
G=-D \frac{\kappa_{0}+\mathrm{i} k_{0}}{\kappa_{0}-\mathrm{i} k_{0}} \mathrm{e}^{2 \mathrm{i} k_{0}(a+d)},
$$

and an explicit expression for $D$ reads

$$
\begin{aligned}
D & =-4 \mathrm{i} \kappa_{0} k\left(\kappa_{0}-\mathrm{i} k_{0}\right) \mathrm{e}^{-\mathrm{i} k_{0} d} /\left[\left(\kappa_{0}^{2}-k_{0}^{2}\right)\left(\kappa_{0}-\mathrm{i} k\right)\right. \\
& \times\left(1-\mathrm{e}^{2 \mathrm{i} k_{0} a}\right) \mathrm{e}^{\kappa_{0} d}-\left(\kappa_{0}^{2}+k_{0}^{2}\right)\left(\kappa_{0}+\mathrm{i} k\right) \mathrm{e}^{-\kappa_{0} d} \\
& \left.\times\left(1-\mathrm{e}^{2 \mathrm{i} k_{0} a}\right)-2 \mathrm{i} \kappa_{0} k_{0}\left(\kappa_{0}-\mathrm{i} k\right)\left(1+\mathrm{e}^{2 \mathrm{i} k_{0} a}\right) \mathrm{e}^{\kappa_{0} d}\right] .
\end{aligned}
$$

The magnitude of $|\psi(\bar{x})|^{2}$ determines the probability to find a quasi-localized in QW electron at some energy $E$, where $\bar{x} \simeq d+a / 2$ is an expected localization maximum of this probability. This probability presented as a function of energy is shown in Fig. 3. It has a sharp resonance for the energy $E$ corresponding to quasi-discrete level of the ground state in QW.

\section{Conclusions}

We have studied spin-dependent electronic transport through the tunneling barrier in a multilayer system with the semiconductor quantum well. We concentrate on the equilibrium state of system assuming that there is no electric current. The splitting of the energy level in the quantum well is related to the different hopping of spinpolarized electrons through the barrier. Using the parameters of $\mathrm{ZnMnBeSe} / \mathrm{ZnSe} / \mathrm{ZnBeSe}$ structure we calculated the dependence of location of dicrete or quasidiscrete level on the parameters of magnetization of electrodes and on the width of tunneling barrier.

\section{Acknowledgments}

This work is supported by the National Science Center in Poland under project of international cooperation "Harmonia" No. DEC-2012/06/M/ST3/00042.

\section{References}

[1] R. Tsu, L. Esaki, Appl. Phys. Lett. 22, 562 (1973).

[2] P.J. Price, Phys. Rev. B 38, 1994 (1988).

[3] Th. Gruber, M. Keim, R. Fiederling, G. Reuscher, W. Ossau, G. Schmidt, L.W. Molenkamp, A. Waag, Appl. Phys. Lett 78, 1101 (2001).

[4] P. Havu, N. Tuomisto, R. Väänänen, M.J. Puska, R.M. Nieminen, Phys. Rev. B 75, 235301 (2005).

[5] N. Lebedeva, P. Kuivalainen, Phys. Status Solidi B 242, 1660 (2005).

[6] C. Ertler, J. Fabian, Phys. Rev. B 75, 195323 (2007).

[7] Y. Song, H. Dery, Phys. Rev. Lett. 113, 047205 (2014).

[8] Z.L. Fang, P. Wu, N. Kundtz, A.M. Chang, X.Y. Liu, J. Furdyna, Appl. Phys. Lett. 91, 022101 (2007).

[9] I. Zutić, J. Fabian, S. Das Sarma, Rev. Mod. Phys. 76, 323 (2004).

[10] S. Yuasa, T. Nagahama, A. Fukushima, Y. Suzuki, K. Ando, Nature Mater. 3, 868 (2004).

[11] R. Meservey, P.M. Tedrow, Phys. Rep. 238, 173 (1994).

[12] S.S.P. Parkin, C. Kaiser, A. Panchula, P.M. Rice, B. Hughes, M. Samant, S.H. Yang, Nature Mater. 3, 862 (2004).

[13] R. Jansen, B.C. Min, S.P. Dash, Nature Mater. 9, 133 (2010).

[14] K.R. Jeon, B.C. Min, A. Spiesser, H. Saito, S.C. Shin, S. Yuasa, R. Jansen, Nature Mater. 13, 360 (2014).

[15] R. Jansen, Nature Mater. 11, 400 (2012).

[16] A. Slobodskyy, C. Gould, T. Slobodskyy, C.R. Becker, G. Schmidt, L.W. Molenkamp, Phys. Rev. Lett. 90, 246601 (2003).

[17] S. Maximov, T. Slobodskyy, A. Gröger, F. Lehmann, P. Grabs, L. Hansen, C.R. Becker, C. Gould, G. Schmidt, L.W. Molenkamp, Semicond. Sci. Technol. 19, 946 (2004).

[18] M.K. Kneip, D.R. Yakovlev, M. Bayer, T. Slobodskyy, G. Schmidt, L.W. Molenkamp, Appl. Phys. Lett. 88, 212105 (2006).

[19] A. Slobodskyy, C. Gould, T. Slobodskyy, G. Schmidt, L.W. Molenkamp, D. Sánchez, Appl. Phys. Lett. 90, 122109 (2007). 